\title{
Neutron detection with cryogenics and semiconductors
}

\author{
Zane W. Bell ${ }^{* 1}$, D. A. Carpenter ${ }^{2}$, S. S. Cristy ${ }^{2}$, V. E. Lamberti ${ }^{2}$, Arnold Burger ${ }^{3}$, \\ Brian F. Woodfield ${ }^{4}$, Thomas Niedermayr ${ }^{5}$, I. Dragos Hau ${ }^{5}$, Simon E. Labov ${ }^{5}$, \\ Stephan Friedrich ${ }^{5}$, W. Geoffrey West ${ }^{6}$, Kenneth R. Pohl ${ }^{7}$, and Lodewijk van den Berg ${ }^{7}$ \\ ' Oak Ridge National Laboratory, 1 Bethel Valley Rd., Oak Ridge, TN 37831-6010, USA \\ ${ }^{2}$ Y-12 National Security Complex, Bear Creek Rd., Oak Ridge, TN 37831-8084, USA \\ ${ }_{3}^{3}$ Fisk University, 1000 Seventeenth Ave. North, Nashville, TN 37208-3051, USA \\ ${ }^{4}$ Brigham Young University, Department of Chemistry and Biochemistry, Provo, UT 84602, USA \\ ${ }_{5}^{5}$ Lawrence Livermore National Laboratory, 7000 East Ave. L-270, Livermore, CA 94550, USA \\ ${ }^{6}$ University of Michigan, 2355 Bonisteel Blvd., Ann Arbor, MI 48109-2104, USA \\ ${ }^{7}$ Constellation Technology Corporation, 7887 Bryan Dairy Rd. Suite 100, Largo, FL 33777-1452, USA
}

Received 8 September 2004, accepted 10 February 2005

Published online 10 March 2005

PACS 29.30.Hs, 29.40.Vj, 29.40.Wk

The common methods of neutron detection are reviewed with special attention paid to the application of cryogenics and semiconductors to the problem. The authors' work with LiF- and boron-based cryogenic instruments is described as well as the use of CdTe and $\mathrm{HgI}_{2}$ for direct detection of neutrons.

() 2005 WILEY-VCH Verlag GmbH \& Co. KGaA, Weinheim

1 Introduction Advances in materials and methods have enabled the detection of radiation by means today that would have seemed, to pioneers in the field a century ago, like science fiction. Improvements in technology have resulted, for gamma ray detection, in high-purity germanium operating at $77 \mathrm{~K}$ and providing $0.1 \%$ energy resolution above $1 \mathrm{MeV}$, more than an order of magnitude improvement over what was (and still is) achievable by scintillators. However, operating below $1 \mathrm{~K}$, cryogenic calorimeters have been used in X-ray astronomy, in the search for dark matter, and more recently in gamma ray spectroscopy, and have achieved better than $70 \mathrm{eV}$ resolution at $60 \mathrm{keV} \mathrm{[1],} \mathrm{a} \mathrm{factor} \mathrm{of} 4$ to 5 improvement over what can be achieved by germanium at that energy. Meanwhile, at the other end of the temperature spectrum, the development of new, wide band-gap semiconductors has sparked research in room temperature gamma ray detectors and has held out the hope of $1-2 \%$ resolution and freedom from cryogenics $[2,3]$.

With such results being reported from the X- and gamma ray world it is natural to examine the possibilities for neutron detection. A cryogenic neutron detector would operate by detecting the heat pulses caused by neutron capture and scattering, while a semiconducting detector would detect the nuclear reaction products from a sensitizer (for example, fission fragments detected in a ${ }^{235} \mathrm{U}$-coated $\mathrm{Si}$ diode) or from some constituent of the semiconductor.

In the following sections, the common methods of neutron detection are described and their deficiencies with respect to neutron spectroscopy at energies above thermal $(0.025 \mathrm{eV})$ are outlined. Published work on neutron-detecting cryogenic calorimeters will be reviewed and work by the present authors on boron- and lithium-based instruments will be discussed. Turning to semiconductors, we review work with coated and native (uncoated) semiconductor, including $\mathrm{Cd}_{1-\mathrm{x}} \mathrm{Zn}_{\mathrm{x}} \mathrm{Te}$ (CZT) and $\mathrm{HgI}_{2}$, as applied to neutron detection. Results obtained by the authors with $\mathrm{HgI}_{2}$ will be shown.

" Corresponding author: e-mail: bellzw@ornl.gov, Phone: +1 865574 6120, Fax: +1 8655768380 
2 Review of neutron detection techniques Since neutrons are uncharged, they are always detected via their nuclear interactions, examples of which are $(n, f),(n, \gamma),(n, \alpha)$, and (n, n'). In all cases it is the interaction of energetic charged reaction products and/or electromagnetic radiation with a detecting medium that produces the sensible signal, typically, but not exclusively, some combination of electric charge or scintillation light.

Not all reaction mechanisms lend themselves to spectroscopy. That is, they are useful as the basis of a detector, but cannot easily be used to determine the energy of the incident neutrons. Reactions that produce fission or the emission of energetic gamma rays result in incomplete registration of the energy of the incident neutron because reaction products escape the detector. For example, fission is accompanied by the emission of $6-8 \mathrm{MeV}$ in prompt and delayed fast neutrons and a similar amount of energy as gamma rays, implying that a fission detector always underestimates the total energy released in the reaction. In addition, fission detectors are not sensitive to the variation of fission $Q$-value with the atomic numbers and masses of the fission product pair.

2.1 Devices based on charged particles Neutron reactions that result in charged particles include fission, recoils (protons and heavier particles), and conversion electrons. The neutron-sensitive layer may be realized as a converter in close proximity to a charged particle detector, or the detecting medium itself (as in the case of ${ }^{3} \mathrm{He}$ ). Included in this category of device are scintillators which convert the energy of the charged particle to light. A discussion of detectors that rely on capture leading to alpha or beta activity is deferred to the following section.

An example of a coated detector is given by Litovchenko et al. [4]. In this instance, a $0.15 \mathrm{~mm}$ thick film of uranium oxide, enriched to $99.92 \%{ }^{235} \mathrm{U}$, was deposited on an aluminium planchet, and placed in contact with a silicon surface-barrier detector. Both alpha particles from the natural decay of uranium and neutron-induced fission fragments were detected by the $100 \mathrm{~mm}^{2}$ silicon diode.

The thermal neutron fission cross section of ${ }^{235} \mathrm{U}$ is 585 barns [5], making their mean free path in a layer of oxide (not specified by Litovchenko, but perhaps $\mathrm{U}_{3} \mathrm{O}_{8}$ ) approximately $1.1 \mathrm{~mm}$ in fully dense material. Consequently, thermal neutrons are absorbed almost uniformly throughout the layer, although only fission fragments born within 10 micrometers of the diode have a chance of reaching it. These fragments, traversing a continuum of paths, reach the diode with a continuum of energies, and create charge within the silicon in proportion to their energy. The electric field within the diode structure collects this charge and external electronics converts it to a voltage pulse whose amplitude is indicative of the fragment's energy. Because of conservation of momentum, only one of the two fission fragments from each fission can enter the diode.

Uranium is not the sole converter material that has been used and silicon is not the only charged particle detector that has been demonstrated. Filho et al. [6] describe a silicon diode covered with boron or polyethylene. In the case of boron, alpha particles and lithium ions are produced upon absorption of thermal neutrons, while the polyethylene generates protons from $(\mathrm{n}, \mathrm{p})$ scattering. In a conceptually similar arrangement, McGregor et al. replaces the silicon diode with GaAs [7], and adds texture to the surface of a GaAs device to improve the efficiency of detection of ions from the ${ }^{10} \mathrm{~B}(\mathrm{n}, \alpha)$ reaction [8].

Aoyama et al. [9] report a device based on a $\mathrm{Gd}$ foil viewed by a silicon diode. The diode was not sensitive to capture gamma rays or resulting X-rays, but, because of the 25 micrometer thickness of the foil, could not distinguish between conversion electrons from the foil (a consequence of the de-excitation of ${ }^{158} \mathrm{Gd}$ proceeding via low-energy E2 transitions) and photo- or Compton electrons. Consequently, Aoyama added a tin foil viewed by a second diode by which to estimate the photon response of the Gd foil.

Coated detectors suffer from the necessity for sensitivity to be balanced against the range $(<5 \mathrm{mi}-$ crometers for the heavy ions, up to $1 \mathrm{~mm}$ for energetic protons) of the reaction products, and ultimately the range of the particles limits the maximum sensitivity. These detectors are necessarily inefficient because the thickness of the converter layer must be sufficiently thin that particles can exit it with sufficient energy to be registered. For a fission-fragment device, typical thickness of the uranium layer is only a 
few micrometers (metal equivalent), making the devices approximately $0.3 \%$ efficient. Litovchenko's detector was quoted at $0.055 \%$, implying an oxide layer containing less uranium than 1 micrometer of pure metal. However, McGregor reports detection efficiency near $4 \%$ with a textured boron-coated diode, and predicts efficiencies approaching $10 \%$ to be possible.

If the neutron-sensitive material is part of the detecting medium itself, issues of range of reaction products disappear since they are created and completely absorbed in the detecting medium. Such is the case for ${ }^{3} \mathrm{He}$ - and ${ }^{10} \mathrm{BF}_{3}$-filled proportional counters. In these devices, the charged particles are generated in the gas as a result of the capture of neutrons, and the ionization created along their paths is collected. Since the energy shared by the charged particles is the sum of the $Q$-value of the capture reaction and the energy of the incident neutron, measurement of the charge is indicative of the energy of the incident neutron. Similarly, boron, lithium, or gadolinium may be incorporated in scintillators in which the energetic charged particles generate light in proportion to the energy deposited by the particles.

Knitel et al. [10] report results for a number inorganic scintillators containing various combinations of $\mathrm{Li}, \mathrm{B}$, and $\mathrm{Gd}$. In most cases, the light yields are disappointingly low $\left(<10^{4}\right.$ scintillation photons $/ \mathrm{MeV}$, electron equivalent) making them unattractive as detector materials. However, cerium activated $\mathrm{YBO}_{3}$, $\mathrm{GdBO}_{3}$, and $\mathrm{Li}_{6} \mathrm{Gd}\left(\mathrm{BO}_{3}\right)_{3}$ are reported to be reasonably bright and emit at 390 and $415 \mathrm{~nm}$.

Organic scintillators consist (generally) of a hydrocarbon plastic, such as polystyrene or polyvinyltoluene, or liquid, such as benzene, xylene, or toluene, into which are dissolved one or more phosphors. Silicone rubber $[11,12]$ has also been demonstrated to be a suitable matrix. Since the phosphors are themselves molecules containing phenyl rings (such as diphenyl oxazole and anthracene), it is necessary for the hydrocarbon matrix also to contain rings so that the energy deposited by ionization can hop from ring to ring until it finally transfers to a phosphor molecule. Typically, the excited singlet to ground state singlet transition has a time constant of a few nanoseconds and the de-excitation results in light. The process is described by Birks [13], Förster [14], and Scholes [15].

Since the number of scintillation photons is approximately proportional to the amount of energy deposited in the scintillator, and the range of even $\mathrm{MeV}$ protons is only a millimeter, the energy of the recoiling proton can be recovered from a measurement of the amount of light from the event. However, $(n, p)$ scattering is isotropic in the center of momentum frame, and, for a neutron with energy $E_{n}$, this leads to a uniform distribution of recoil proton energies from 0 (grazing collision) to $E_{n}$ (head-on collision). Consequently, it is necessary to unfold a response function from the measured spectrum to obtain the spectrum of incident neutrons.

The unfolding process is a stripping procedure, and it accumulates errors in the low-energy portion of the spectrum. The problem is compounded by the fact that recoil protons produce less light (by a factor of $4-8$, depending on the matrix, phosphors, and energy) than do electrons. Since the noise floor of an uncooled photomultiplier tube is often near $10 \mathrm{keV}$ (electron equivalent), neutrons with energy much below $200 \mathrm{keV}$ do not produce a pulse-height spectrum that can be analyzed with any certainty.

Recently, Robertson et al. [16], have reported the fabrication of a diode from $270 \mathrm{~nm}$ boron carbide layer deposited on n-type Si. They exposed the device to a nuclear reactor, acquired pulse-height spectra, and ascribed features in the spectra to the reaction products of the ${ }^{10} \mathrm{~B}(\mathrm{n}, \alpha)^{7} \mathrm{Li}$ reaction. In subsequent work [17], they report the fabrication of an all-boron-carbide device made by depositing n-type boron carbide onto a p-type boron carbide layer previously deposited on an aluminium substrate, and, on exposure to a $120 \mathrm{n} / \mathrm{cm}^{2} / \mathrm{s}$ thermal neutron flux, they observed a current of approximately $18 \mathrm{fA}$. Unfortunately, the authors give no indication that this current is consistent with the incident flux, nor any explanation of how they observe such small currents in the presence of reported DC bias currents several orders of magnitude larger. Further compounding the issue is the failure of the authors to perform simple experiments to separate the neutron and gamma responses of the devices. It is also possible that some of the observed spectral features are a consequence of fast neutron interactions. This is not to say the devices do not perform as described, but rather that there are some questions regarding the interpretation of the data. 
2.2 Devices based on capture gamma rays and induced radioactivity Neutron capture usually results in the formation of a nucleus in a highly excited state. This state may decay by the emission of charged particles as described above, or, as is more likely, by the emission of a cascade of gamma rays, conceptually similar to the cascade of X-rays emitted when an ion captures an electron. In addition, it is possible for the transmuted nucleus to be unstable and subsequently decay by alpha, beta, or positron emission, or electron capture followed by the emission of more gamma rays and/or X-rays. Examples of these reactions are ${ }^{113} \mathrm{Cd}(\mathrm{n}, \gamma){ }^{114} \mathrm{Cd}$ (stable product) and ${ }^{197} \mathrm{Au}(\mathrm{n}, \gamma){ }^{198} \mathrm{Au}$ (unstable product) The deexcitation can also produce conversion electrons, as in the case of ${ }^{158} \mathrm{Gd}$, described above.

The similarities between devices that detect either capture gamma rays or gamma rays resulting from induced radioactivity from the product are such that it is sufficient to consider them together. In both cases, a gamma detector is placed in proximity to the neutron sensitive material and the resulting pulseheight spectrum is analyzed for evidence of the signature gamma ray or rays.

Bell [18] describes the use of the $478 \mathrm{keV}$ gamma ray that is emitted in the ${ }^{10} \mathrm{~B}(\mathrm{n}, \alpha)^{7} \mathrm{Li}^{*}$ reaction. The use of boron as a converter generating charged particles has been described above, however if the converter is placed close to an efficient gamma ray detector, such as $\mathrm{CdWO}_{4}$, the gamma ray can be used as a signature of the presence of thermal neutrons. Although this is a significantly less efficient method than direct detection of charged particles, the limitations imposed by the fabrication considerations of semiconductor detectors are removed by the use of scintillator that is readily fabricated in large volume and surface area. Consequently, in a flux-starved environment, higher count rates may be achieved at lower total cost because of the larger sensitive volume of a scintillator. Other materials with high neutron capture cross section that produce an analyzable spectrum are $\mathrm{Cd}$ and $\mathrm{Hg}$, both of which will be discussed below.

As the name implies, activation foil analysis relies on the detection of induced radioactivity in a detecting medium. Generally, this technique is not useful for single event counting, but rather is useful only to determine the presence of a flux. The method is hindered by the time needed to activate the foil (long half-life foil implies long time to reach saturation), the loss of activity resulting from delays in reaching the counting apparatus, and from self-absorption effects when the induced radioactivity manifests itself as alpha particles, low-energy beta particles, or X-rays.

Johnson [19] reported the detection of induced radioactivity in a CdS crystal serving as both neutron absorber and detector. Subsequent to irradiation by $14 \mathrm{MeV}$ neutrons $\left(10^{11}-10^{17} \mathrm{n} / \mathrm{cm}^{2}\right)$, the decays of ${ }^{115} \mathrm{Cd}$ and ${ }^{32} \mathrm{P}$ were observed. CdS is inappropriate for thermal neutron activation detection since the sulphur cross section is very small, and the dominant thermal neutron absorber among the cadmium isotopes, ${ }^{113} \mathrm{Cd}$, leads to a stable product.

Some activation foil materials, such as rare earth elements, can be incorporated into scintillators, and Bell et al. [20] show a method for doing so. Figure 1 shows a spectrum obtained immediately after the irradiation of plastic scintillator containing the tributylphosphate complex of $\mathrm{Dy}\left(\mathrm{NO}_{3}\right)_{3}$. The counting time was 400 seconds starting within 20 seconds after the end of neutron irradiation. The scintillator sample was irradiated for 10 minutes to saturate the activity of the $108 \mathrm{keV}$ excited state of ${ }^{165} \mathrm{Dy}$.

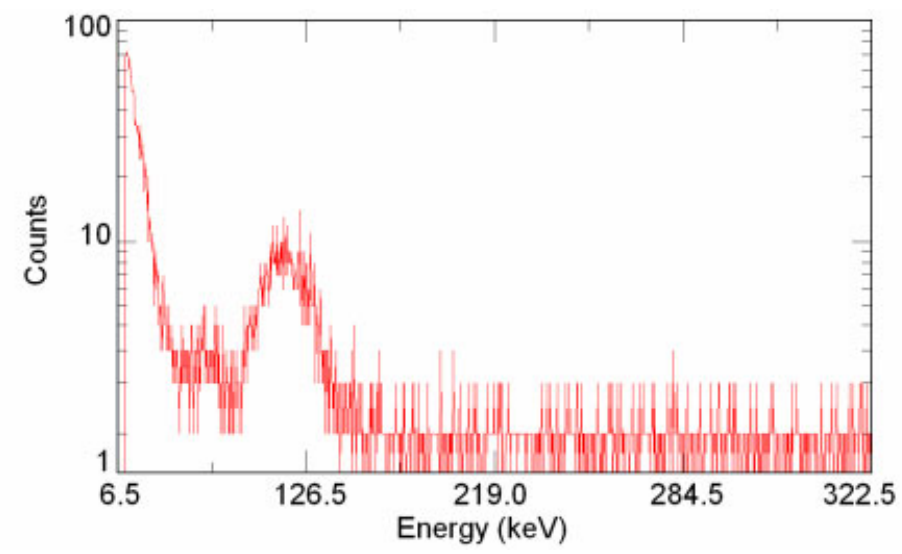

Fig. 1 Spectrum of activated Dy contained within plastic scintillator. The peak near $126 \mathrm{keV}$ is from the 75 second decay of the $108 \mathrm{keV}$ first excited state of ${ }^{165}$ Dy and is caused by the ejection of L-shell electrons. 
Since the Dy is uniformly distributed within the scintillator, the low energy electrons generated in the decay deposit all their energy within the scintillator, making a self-absorption correction unnecessary.

Activation foils can be used for spectroscopy by taking advantage of the thresholds for various reactions. Knoll [21] provides a list of candidates for threshold activation analysis but a perusal of the threshold energies and the magnitude of the relevant cross sections quickly persuade the reader that precise spectroscopy is not feasible by this technique.

2.4 Devices based on radiation damage Detectors in this category are the track-etch devices and bubble dosimeters. The track-etch devices use an insulating material that is damaged by the passage of ionizing radiation, which must be heavy particles. Tracks are "developed" by etching the detecting medium in an acid or base and take advantage of the fact that undamaged material is removed more slowly than damaged material. However, if the angle of incidence of the radiation is too great, then the etch rate perpendicular to the surface will exceed the rate along the track and the track will not be enhanced. Neutrons are detected with these devices by placing a hydrogenous radiator in front of the track-etch material to detect recoil protons, by making the detector with a plastic and using the hydrogen constituent, or by placing the track-etch material near fissionable material, or by incorporating fissionable material in the track-etch medium to detect fission fragments. Although the use of fissionable materials in this application does not lend itself to spectroscopy, measuring the distribution of the lengths of proton recoil tracks provides analogous information to that which is obtained from hydrogenous scintillators.

Bubble dosimeters operate on the principle that radiation damage in a superheated fluid provides nucleation sites for bubble formation. The number of bubbles that form is a function of the incident neutron energy and flux, and the temperature and pressure under which the fluid is kept. In addition, the threshold energy for bubble formation depends on the temperature and pressure of the fluid. By preparing a set of dosimeters with varying degrees of superheating, crude spectroscopy can be performed.

3 Cryogenic detectors Cryogenic detectors operate by detecting the heat pulse generated by the interaction of a neutron with the absorber. For a material with heat capacity $C$, the temperature rise due to the absorption of energy $\Delta E$ is given by

$$
\Delta T=\frac{\Delta E}{C} .
$$

The heat capacity of the absorber must be adjusted by a combination of the proper material and operating temperature so that the absorption of the interaction energy yields a detectable temperature rise that remains within the operational envelope of the detector. From knowledge of $C$, and the measurement of $\Delta T$, the absorbed energy may be recovered via Eq. (1).

The resolution of a cryogenic detector, in contrast to a solid-state detector, does not depend on the energy deposited in the absorber. Moseley et al. [22], have shown that in the simplest case of a single absorber/detector thermal unit the distribution of pulses corresponding to monoenergetic radiation has theoretical full width at half maximum given by

$$
F W H M=2.35 \xi \sqrt{\left(k_{B} T\right)^{2}\left(C / k_{B}\right)},
$$

with $\xi$ being a factor numerically between 1 and 2 that depends on the absorber and temperature sensor. Equation (2) implies that for insulators following the Debye model, $F W H M$ decreases as $T^{5 / 2}$ at temperatures below $1 \mathrm{~K}$, making low operating temperature highly desirable.

What nuclear reactions are appropriate for a cryogenic spectroscopic detector? From the discussion in Section 2, the most appropriate reactions are those producing only charged particles, few or no gamma rays, and having high absorption cross section. In such cases, the energy of the neutron is recovered from Eq. (1) by subtracting the $Q$-value of the capture reaction from the calculated energy deposition. Examination of the nuclear data (Ref. [5], for example) narrows the candidate absorbers to ${ }^{3} \mathrm{He},{ }^{10} \mathrm{~B}$, and ${ }^{6} \mathrm{Li}$, and, of these, ${ }^{3} \mathrm{He}$ is not practical because it is an inert gas at room temperature and a mechanically stable absorber would be extremely difficult to fabricate. 
De Marcillac et al. [23], reports the detection of alpha particles from a variety of sources (these are used because their range in LiF is only an order of magnitude smaller than the range of thermal neutrons) and from thermal neutron capture in a 2 -gram LiF crystal held at $80 \mathrm{mK}$. Temperature was sensed with thermistors, and the authors report $16 \mathrm{keV}$ resolution at $4.8 \mathrm{MeV}$ deposited energy. They did not perform measurements with fast neutrons, but, based on calibration with alpha particles and thermal neutrons, they concluded that the response of the crystal was different when $4.78 \mathrm{MeV}$ was deposited by an alpha and triton rather than by a single alpha particle.

Silver et al. [24] report results obtained with a series of ${ }^{6} \mathrm{LiF}$ crystals ranging in mass from approximately $80 \mathrm{mg}$ to $2.6 \mathrm{~g}$, with the best resolution of $39 \mathrm{keV}$ obtained with the $80 \mathrm{mg}$ crystal operating at $323 \mathrm{mK}$. They exposed the $2.6 \mathrm{~g}$ crystal to neutrons and were able to observe pulses caused by thermal, 3.996, 5.167, and 7.223 MeV neutrons, the high-energy neutrons having been obtained from a D-D accelerator source. The temperature sensor was a neutron transmutation doped p-type Ge thermistor.

Richardson et al. [25, 26], and Chowduri et al. [27] describe the use of Li alloys with $\mathrm{Pb}$ and $\mathrm{Mg}$, respectively, for use as flux monitors. In these particular instances, the goal of the work was to develop a device for precise measurement $\left(0.1 \%\right.$ accuracy) of thermal and sub-thermal fluxes. Using ${ }^{6} \mathrm{LiPb}$ alloy, Richardson et al., first demonstrated a dual-compensated calorimeter (temperature of neutron absorber and heat bath independently controlled) with a $5.7 \mathrm{~cm}$ diameter by $6.3 \mathrm{~mm}$ thick absorber sensitive to a thermal current of $1.3 \times 10^{6} \mathrm{n} / \mathrm{s}$, and in their subsequent paper, estimate the sensitivity of an improved design to be a factor of $1300 \mathrm{n} / \mathrm{s}$. Chowduri investigated ${ }^{6} \mathrm{Li}_{0.76} \mathrm{Mg}_{0.24}$ in place of the LiPb alloy and reported sensitivity to neutron currents of $10^{5} \mathrm{n} / \mathrm{s}$.

Takahashi et al. [28], propose a detector based on superconducting $\mathrm{MgB}_{2}$ formed in a thin serpentine strip on a sapphire substrate. The basic scheme is to monitor the resistance of the $\mathrm{MgB}_{2}$ strip while it is irradiated by neutrons. With strips having cross sections of $1 \mu \mathrm{m}^{2}$, the absorption of the reaction products from the ${ }^{10} \mathrm{~B}(\mathrm{n}, \alpha)$ would be expected to cause sufficient local heating for a small volume of the strip to become normal-conducting. However, the authors indicated that they were only in the preliminary design stages of the project and did not present count rate or pulse-shape data.

Nakamura et al. [29], report that they have fabricated a neutron detector from a $0.3 \mathrm{~mm}$ thick $\mathrm{Li}_{2} \mathrm{~B}_{4} \mathrm{O}_{7}$ single crystal made from natural materials and read out with superconducting tunnel junctions. The crystal was held at $450 \mathrm{mK}$ and exposed to both gamma and neutron sources. As expected, the device showed little efficiency for gamma rays, but, oddly, did not show peaks corresponding to capture by lithium and boron. In another paper [30], Nakamura et al., immersed an In-Sb semiconductor in ${ }^{3} \mathrm{He}$ gas held at $4.2 \mathrm{~K}$ to detect reaction products from the ${ }^{3} \mathrm{He}(\mathrm{n}, \mathrm{t})$ reaction. Although operated at cryogenic temperatures, the device is more properly classified as a coated semiconductor detector similar in operation to the coated diodes described in Section 2.1.

The present authors have recently reported [31] initial results with a bolometer read out by a transition edge sensor (TES), a very sensitive thermometer held between the normal and superconducting state, whose current is sensed by a SQUID. A small rise in temperature results in a large change in TES resistance, which in turn results in a large change in current in the bias circuit. This changing current passes through a coil, and changes the magnetic field sensed by the SQUID, which is in the negative feedback loop of an amplifier, and permits the measurement of small currents in low impedance circuits.

The structure of the absorber is shown in Fig. 2, below; the reader should be aware that this is a proofof-principle device intended to demonstrate a TES/SQUID instrument with a boron-containing absorber. The absorber is glued to the TES which has been deposited directly onto a silicon nitride membrane grown on a silicon wafer. After the silicon nitride is deposited, the silicon wafer is etched away to reduce the thermal conductivity between the TES and the silicon wafer (which is coupled to the cold bath). The dimensions of the absorber are approximately $1 \mathrm{~mm}^{2}$ by $0.25 \mathrm{~mm}$ thick. The refrigerator is described by Friedrich et al. [32].

The TES is a Mo/Cu multilayer with R-T characteristics as shown in Fig. 3. Noting that the width of the transition region is only a few tenths of a $\mathrm{mK}$, and knowing that the energy deposited in a boron- or lithium-based absorber is $2-5 \mathrm{MeV}+E_{n}$, it is easily seen that the absorber's heat capacity must be ap- 
proximately $10 \mathrm{pJ} / \mathrm{mK}(60 \mathrm{MeV} / \mathrm{mK})$ for the TES to remain in the linear portion of the transition region. In addition, the thinness of the $\mathrm{Si}_{3} \mathrm{~N}_{4}$ membrane requires the absorber to have a mass of only a few $\mathrm{mg}$. Among the boron compounds, $\mathrm{TiB}_{2}$, a normal conductor, has the required characteristics.
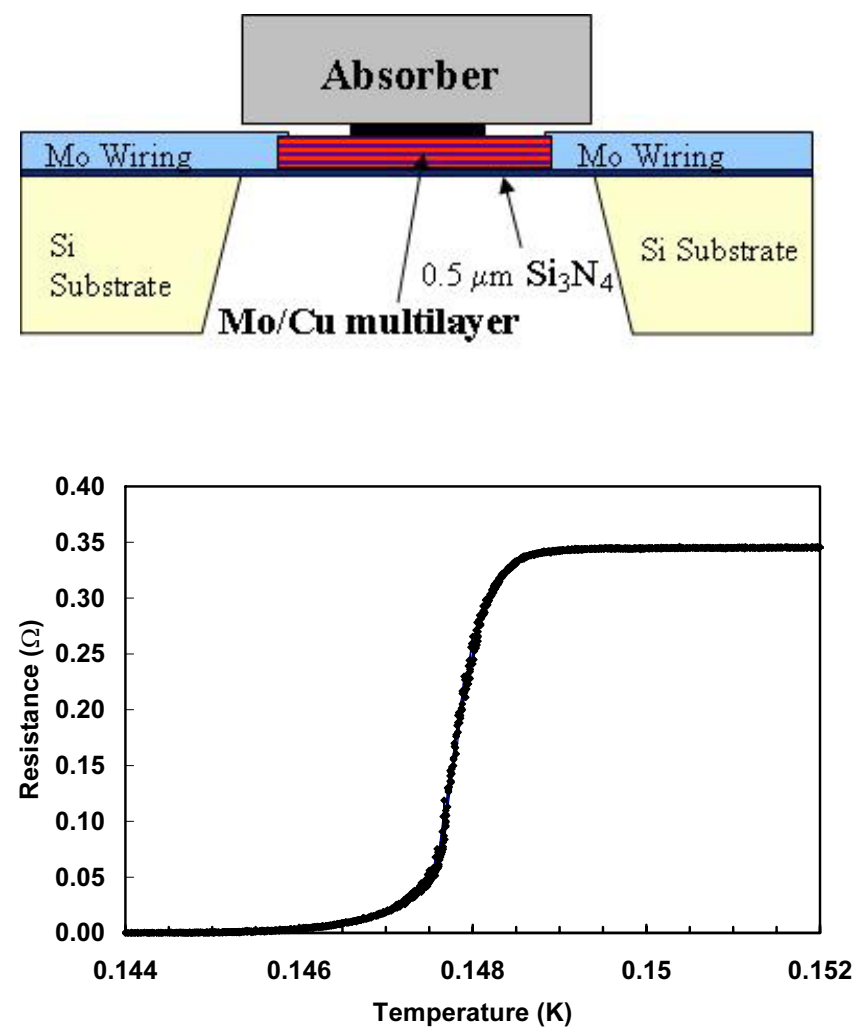

Fig. 2 Schematic of arrangement of neutron absorber and $\mathrm{Mo} / \mathrm{Cu}$ TES on $\mathrm{Si}_{3} \mathrm{~N}_{4}$ membrane. The drawing is not to scale.

Fig. 3 Variation of resistance of TES as a function of temperature near the transition. Note that the transition occurs in only a few tenths millikelvin, making the sensitivity approximately $1000 \Omega / \mathrm{K}$ in the transition region.

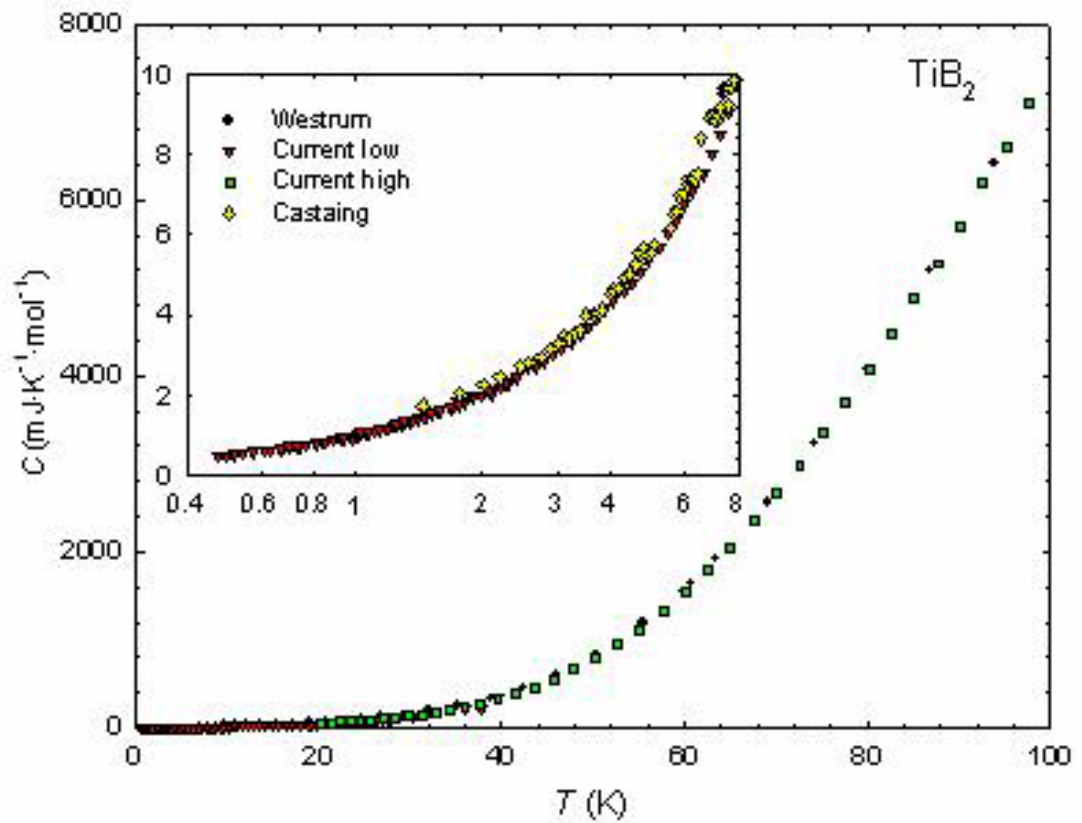

Fig. 4 Heat capacity of $\mathrm{TiB}_{2}$ as measured in this work and compared to earlier measurements. The inset shows the data from 500 $\mathrm{mK}$ to $8 \mathrm{~K}$ in greater detail. 
The heat capacity of $\mathrm{TiB}_{2}$, as measured in this work between $500 \mathrm{mK}$ and $100 \mathrm{~K}$ and compared to data from the literature [33, 34], is shown in Fig. 4. The low temperature data is well fit by the Debye model alone or with the addition of a Schottky anomaly ascribable either to the boron or to the presence of impurities. Extrapolating the Debye fit to $100 \mathrm{mK}$ (the desired operating temperature), results in a predicted heat capacity of $0.100 \mathrm{~mJ} / \mathrm{K} / \mathrm{mol}$. If a Schottky anomaly is hypothesized, then the two models (boron alone or impurities) lead to predicted heat capacities of 0.435 and $0.408 \mathrm{~mJ} / \mathrm{K} / \mathrm{mol}$ at $100 \mathrm{mK}$, respectively. In the impurity model, an entirely plausible concentration of $450 \mathrm{ppm}$ fit the data.

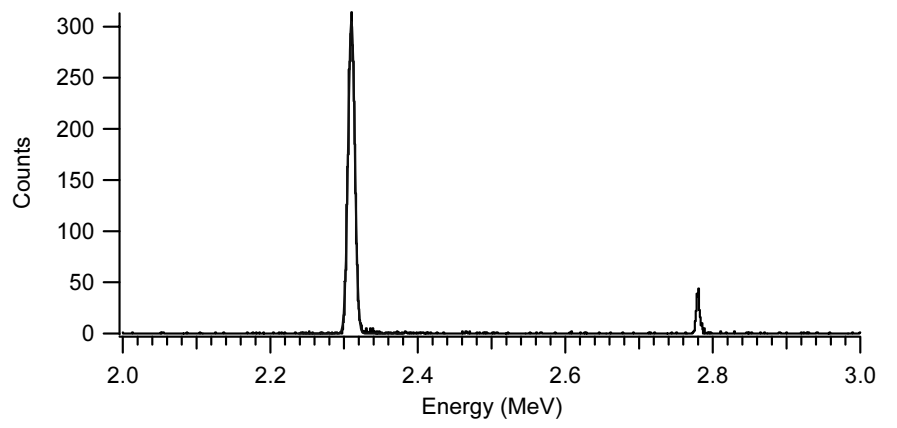

Fig. 5 Spectrum of pulses from $\mathrm{TiB}_{2}$ absorber irradiated with thermal neutrons. The two peaks are from disintegrations leaving $\mathrm{Li}$ in its ground state and first excited state.

Figure 5 shows a spectrum obtained from a thermalized ${ }^{252} \mathrm{Cf}$ source using a TES/SQUID bolometer with a $4 \mathrm{mg}(\sim 12 \mathrm{pJ} / \mathrm{mK}) \mathrm{TiB}_{2}$ absorber in the configuration of Fig. 2. The two peaks have areas in the ratio of $14.86: 1$, exactly what would be expected from the ${ }^{10} \mathrm{~B}(\mathrm{n}, \alpha)$ reaction at thermal energies. The width of the $2.31 \mathrm{MeV}$ peak is $10.5 \mathrm{keV}$ while that of the $2.79 \mathrm{MeV}$ peak is $5.5 \mathrm{keV}$, the discrepancy being caused by the emission of a $478 \mathrm{keV}$ gamma ray from the decay of excited ${ }^{7} \mathrm{Li}$ nuclei in flight. This introduces an uncertainty in deposited energy of $9.8 \mathrm{keV}$, and if the instrument's resolution is assumed to be $5.5 \mathrm{keV}$, then when the $9.8 \mathrm{keV}$ is added in quadrature, the observed resolution is obtained.

While a small absorber is sufficient for thermal neutrons, the $1 / \mathrm{v}$ dependence of the boron and lithium cross sections demand that absorbers with masses of approximately 10 grams be used. Consequently, a holder to accommodate absorbers with volumes of $15 \mathrm{~cm}^{3}$ has been designed, and is shown in Fig. 6 .

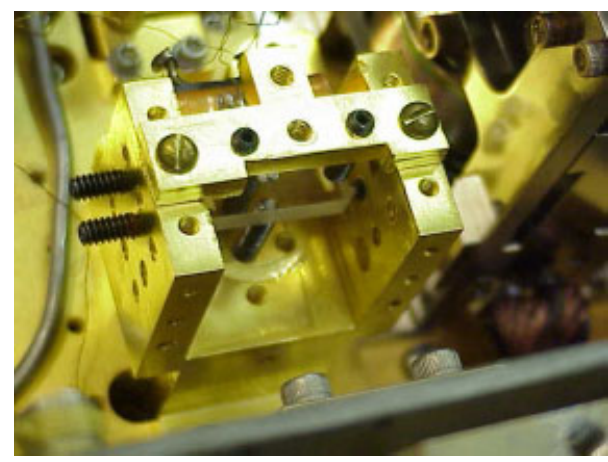

Fig. 6 Large-absorber holder with $0.8 \mathrm{~cm}^{3}$ nat $\mathrm{LiF}$ crystal. The crystal is supported on thermally insulating balls mounted on screws, two of which are visible at the left and one is visible under the crystal.

In this configuration, the arrangement of Fig. 2 was inverted and the weak thermal link between the TES and cold bath was realized with thin wires. It should be noted that while the holder is at $100 \mathrm{mK}$, the absorber's supports are thermally insulating so that heat generated by the absorption of neutrons must flow through the glue to the TES and then through the contact wires that are maintained at the temperature of the bath. A spectrum obtained in this configuration is shown in Fig. 7, below. The resolution seen in this spectrum is approximately $45 \mathrm{keV}$ and is thought to be caused positional dependence of the response. The low energy continuum was caused by inelastic scattering of neutrons, and scattering of gamma rays. 


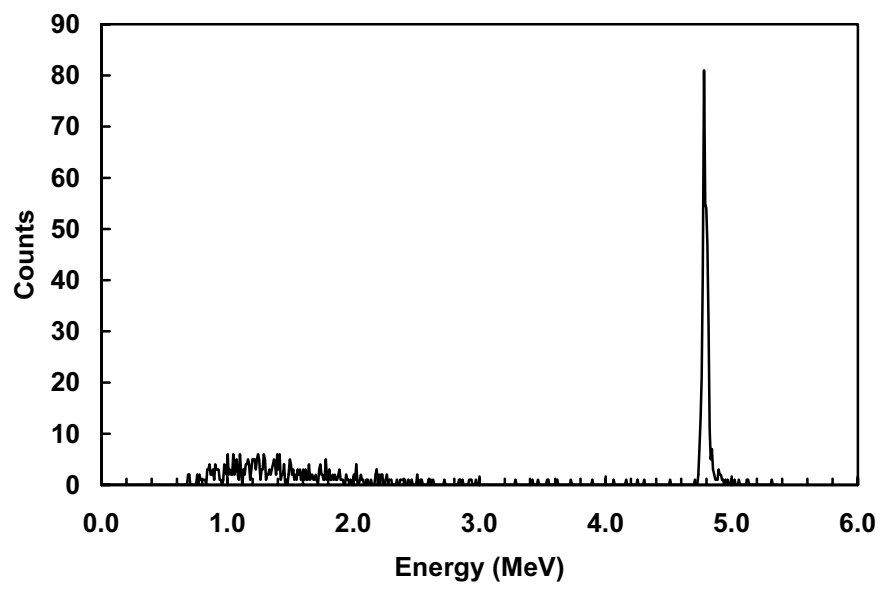

Fig. 7 Spectrum of obtained with $0.8 \mathrm{~cm}^{3}$ ${ }^{\text {nat }} \mathrm{LiF}$ crystal in holder of Fig. 6. Note the high-energy tail in the peak indicative of the presence non-thermal neutrons.

Which will make the better absorber, ${ }^{10} \mathrm{~B}$ or ${ }^{6} \mathrm{Li}$ ? The advantages of ${ }^{6} \mathrm{Li}$ are the higher $Q$-value of the capture reaction $(4.78$ v. $2.79 \mathrm{MeV})$ and the fact that there is only a single available nuclear channel (there are two capture reactions in boron, with Doppler broadening in one; see Fig.5). The higher $Q$ value is important because capture events will be offset by the $Q$-value from scattering events and gamma ray interactions. However, of lithium compounds, only LiF is suitable for use because of its relative inertness and the relatively high density of lithium $(0.064$ atoms $/ \mathrm{b}-\mathrm{cm})$, while there is a myriad of air- and water-stable borides, not to mention elemental ${ }^{10} \mathrm{~B}$ with a density of 0.13 atoms $/ \mathrm{b}-\mathrm{cm}$. In addition, inelastic neutron scattering from fluorine (the threshold for which is $115 \mathrm{keV}$ ) and from ${ }^{6} \mathrm{Li}$ itself (important above $2.5 \mathrm{MeV}$ ) result in escaping gamma rays and neutrons whose now lowered energy makes them more likely to be captured. Unfortunately, capture of these neutrons distorts the spectrum if their coincident gamma rays escape, making the quality of the spectrum from a lithium absorber dependent, then, on the fate of inelastic gammas. In contrast to this, the effects of inelastic scattering in elemental ${ }^{10} \mathrm{~B}$ become important only above $4.4 \mathrm{MeV}$. Therefore, in addition to using Li absorbers, we are investigating the possibility of fusing elemental boron according to the procedure of Holcombe et al. [35], no mean feat, considering that the melting point is approximately $2100 \mathrm{C}$. It should be noted that elastic scattering followed by neutron capture does not distort the spectrum because the ion's recoil energy is completely absorbed in the detector.

4 Semiconductor detectors For the purposes of this paper, the term "semiconductor detector" refers to devices in which the semiconducting material absorbs or interacts with the incident neutron rather than merely detecting reaction products produced externally to the device. That is, coated semiconductors (discussed in Section 2.1, above) will be omitted.

Silicon is useful as a semiconductor detector owing to the ${ }^{28} \mathrm{Si}(\mathrm{n}, \mathrm{p})$ and ${ }^{28} \mathrm{Si}(\mathrm{n}, \alpha)$ reactions, and to the state of the art of manufacturing, enabling the fabrication of devices with surface areas of hundreds of square millimetres. Unfortunately, since the above-mentioned reactions have small cross sections (peak values are $\sim 300 \mathrm{mb}$ [36]), silicon diodes typically are not more than a few $\mathrm{mm}$ thick, and the reactions have thresholds near 3 and $4 \mathrm{MeV}$, respectively, silicon finds application in high flux, high energy environments. The typical interaction rate in $1 \mathrm{~mm}$ can be calculated to be approximately $0.1 \%$ at $12 \mathrm{MeV}$.

Miller and Kavanagh [37] report the use of lithium-drifted silicon diodes with incident neutrons in the energy range $6-17 \mathrm{MeV}$. They were able to identify peaks in the pulse height spectrum from alpha particles leaving ${ }^{25} \mathrm{Mg}$ in its ground state and first four excited states, ${ }^{28} \mathrm{Al}$ in its ground state and first eight excited states (although not all were resolved), and ${ }^{26} \mathrm{Mg}$ in its ground and first excited states. The latter species arises from the ${ }^{29} \mathrm{Si}(\mathrm{n}, \alpha)$ reaction; ${ }^{29} \mathrm{Si}$ making up $4.7 \%$ of the natural. From knowledge of the various cross sections and resolution of the detector, and assuming that all (except for a $31 \mathrm{keV}$ gamma from the first excited state of ${ }^{28} \mathrm{Al}$ ) gamma rays escape, the pulse height spectrum can be un- 
folded to recover the incident spectrum. Dési [38] using the same technique used $\mathrm{Si}(\mathrm{Li})$ detectors and silicon diodes to measure fast fluxes in a reactor.

Chung and Chen [39] have measured neutron fluxes with high purity Ge detectors usually used for gamma ray spectroscopy. The technique takes advantage of the ${ }^{73} \mathrm{Ge}(\mathrm{n}, \gamma),{ }^{72,74} \mathrm{Ge}(\mathrm{n}, \mathrm{n}$ ' $\gamma)$ reactions which produce gamma rays at 596 and $691 \mathrm{keV}$. The authors report that in a $10 \%$ detector $\left(58 \mathrm{~cm}^{3}\right)$, a count rate of 0.5 event/s in the 596 photopeak at an incident flux of $10 \mathrm{n} / \mathrm{cm}^{2} / \mathrm{s}$. However, since this technique measures the gamma ray energies, the recoil energy of the Ge nucleus is lost except for inelastic scattering leading to Doppler broadening of photopeaks.

Diamond as a semiconductor detector has been exploited by Kozlov et al. [40], Kovalchuk, Trotsik, and Kovalchuk [41], Bergonzo et al. [42], and Schmid et al. [43]. In these instances, the authors applied an electric field across a diamond stones or CVD films to collect the charge generated by (n, $\alpha)$ or $(n, n)$ reactions. The very recent work of Schmid et al., shows exceptional results with resolution of $2.9 \%$ at 14 $\mathrm{MeV}$. Below the $(\mathrm{n}, \alpha)$ threshold, however, a diamond detector relies on elastic and inelastic scattering from carbon and so faces the same difficulties that proton recoil detectors at these energies: The spectrum must be unfolded to recover spectroscopic information.

Doty and collaborators have investigated $\mathrm{Li}_{2} \mathrm{~B}_{4} \mathrm{O}_{7}$, and $\mathrm{BaB}_{4} \mathrm{O}_{7}$ [44], hexagonal boron nitride [45], and organic semiconductors [46] for neutron detectors. Semiconducting detectors based on boron carbide have already been discussed in Section 2.1. References [43] and [44] describe devices intended for detection only, while Ref. [45] describes thin film devices that, unless loaded with some material like lithium or boron, will not have sufficient efficiency for spectroscopy applications. In addition, the spectrum developed by an organic semiconductor will require unfolding as described in Section 2.1. Organic semiconductors are still in their infancy with respect to their use as radiation detectors.

CdZnTe and CdTe have also been used for thermal neutron detection. Siffert et al. [47], Vradii et al. [48], Fasasi et al. [49], and McGregor et al. [50] have all used the capture gamma rays from the ${ }^{113} \mathrm{Cd}(\mathrm{n}, \gamma){ }^{114} \mathrm{Cd}$ reaction as a signature of the presence of neutrons, and show that the $558 \mathrm{keV}$ emission is easily seen in small detectors. Vradii concludes from observed count rates that the efficiency of the CdTe devices is comparable to that of an unspecified ${ }^{3} \mathrm{He}$ tube, when the entire pulse-height spectrum is used. Fasasi, however, reports efficiency of about $5 \%$, based on the counts in the entire spectrum, $0.5 \%$ when only the $96 \mathrm{keV}$ gamma is used, $0.38 \%$ when the $558 \mathrm{keV}$ line is measured with a 2 mm thick detector, and $0.28 \%$ when that line is measured with a $1 \mathrm{~mm}$ thick detector. McGregor reports $3.7 \%$ efficiency for the $558 \mathrm{keV}$ gamma ray with a $3 \mathrm{~mm}$ thick CdZnTe detector. It is not obvious that all these measurements are in agreement.

The cadmium reaction may not be the best choice for semiconductor detectors based on capture gamma rays. The de-excitation of ${ }^{114} \mathrm{Cd}$ contains hundreds of gamma rays between $60 \mathrm{keV}$ and $9 \mathrm{MeV}$, with $6(558,661,805,756,725$, and $96 \mathrm{keV})$ having probabilities of emission exceeding $1 \%$. Since these gamma rays are emitted in cascades, the detector sees them as simultaneous, and sums the energy from each deposited in it. Since neutron capture occurs within $1 \mathrm{~mm}$ of the surface of CZT, and the interactions of all the gamma rays are independent of each other, and there are a large number of individual gamma rays, the total amount of energy deposited by each event must resemble a continuum more than a spectrum of discrete peaks, serving to decrease the efficiency as measured by examining the photopeak. If a detector constituent emits only one gamma ray, however, or if the other gamma rays are invisible to the detector, then the signature gamma ray will stand out from the continuum.

Beyerle and Hull [51], and Bell, Pohl, and van den Berg [52] have exploited this property of the ${ }^{199} \mathrm{Hg}(\mathrm{n}, \gamma)$ reaction to detect thermal neutrons. ${ }^{199} \mathrm{Hg}$ comprises $16.9 \%$ of natural mercury, but has a capture cross section of $2200 \mathrm{~b}$ [53]. Since the dominant capture gamma ray from this reaction has energy $368 \mathrm{keV}$ and occurs in $81 \%$ of events and the vast majority of others have energies in excess of $1.5 \mathrm{MeV}$ [54], a thin $\mathrm{HgI}_{2}$ detector would be expected to generate a $368 \mathrm{keV}$ photopeak broadened by the small amount of energy deposited by the other gamma rays. This is exactly what was observed in Refs. [50] and [51].

Bell, Pohl, and van den Berg modified the arrangement of Beyerle and Hull by adding a 100\% absorptive (at $0.0253 \mathrm{eV}$ ) ${ }^{10} \mathrm{~B}$ cladding to the $\mathrm{HgI}_{2}$ detector. The proximity of the boron to the detector ensured 
that the single $478 \mathrm{keV}$ gamma ray from the ${ }^{10} \mathrm{~B}(\mathrm{n}, \alpha)$ reaction had a high probability of entering the $\mathrm{HgI}_{2}$ crystal, and both gamma rays were observed. It should be noted that since the energies of these gamma rays is lower than the main ones from $\mathrm{Cd}$, the probability of gamma ray detection is higher in an $\mathrm{HgI}_{2}$-based device.

Both Cd- and Hg-based devices are thermal neutron detectors; no spectral information for the neutrons can be obtained from measurement of the gamma rays. However, consider an $\mathrm{HgI}_{2}$ detector, with a boron cladding, that has been packed in polyethylene moderator, as shown in Fig. 8. Monte Carlo modelling indicates that crude spectroscopic information over a broad energy range can be extracted from an analysis of the two capture gamma rays.

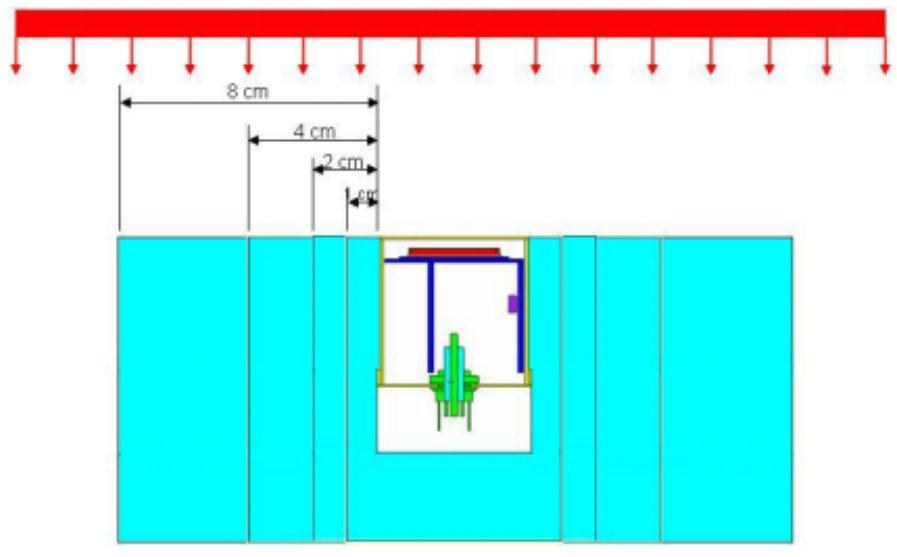

Fig. 8 Schematic of $\mathrm{HgI}_{2}$ detector packed in polyethylene. The $\mathrm{HgI}_{2}$ is the topmost horizontal structure; the boron cladding is not visible, but is on the top surface of the $\mathrm{HgI}_{2}$. The vertical structures are the printed circuit boards and high-voltage connector of the detector. The vertical lines represent the $1,2,4$, and $8 \mathrm{~cm}$ boundaries of polyethylene moderator. There are $4 \mathrm{~cm}$ of polyethylene behind. Neutrons, at constant fluence, are incident from the region above.

Essentially, this configuration is expected to behave like the Bonner spheres and long counter described by Knoll [55]. Figure 9 shows the effects of increasing wall thickness on the calculated photopeak areas as a function of incident neutron energy. The dramatic increase of the number of Hg photopeak $(368 \mathrm{keV})$ events with increasing moderator thickness is indicative of the increase in the number of returning neutrons, while the only modest increase in boron events $(478 \mathrm{keV})$ indicates that the $\mathrm{HgI}_{2}$ layer is not $100 \%$ absorptive. Figure 9 also shows the response, as measured by the ratio of the $478 \mathrm{keV}$ peak to the $368 \mathrm{keV}$ peak, is approximately constant from approximately $10 \mathrm{eV}$ to $10 \mathrm{MeV}$ for the thinnest walls.

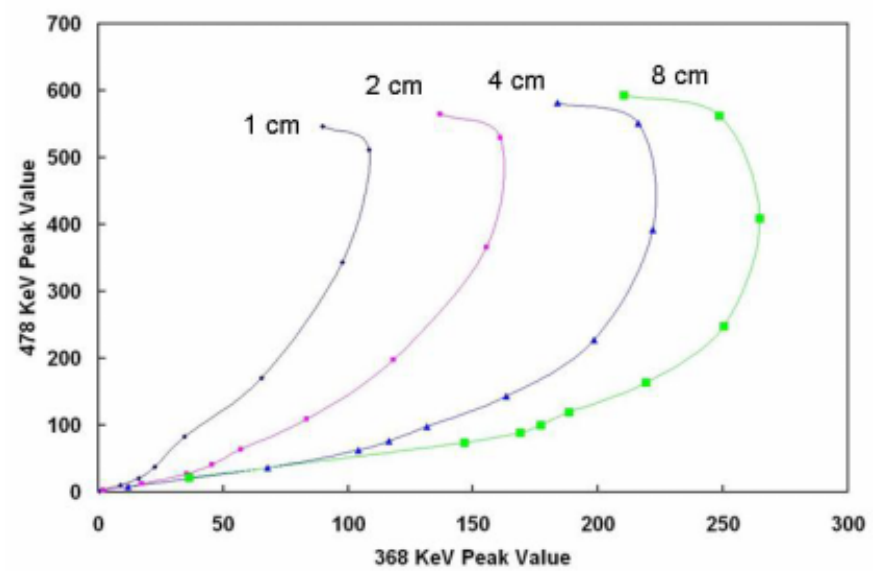

Fig. 9 Calculated [56] locus of points defined by the photopeak heights at $368 \mathrm{keV}$ and $478 \mathrm{keV}$ for a boron-clad $2.6 \mathrm{~mm}$ thick $\mathrm{HgI}_{2}$ detector in the configuration of Fig. 8 . Each labelled line indicates the thickness of the polyethylene side walls. The points along each line represent, clockwise from the top, $0.025 \mathrm{eV}, 0.1 \mathrm{eV}, 1 \mathrm{eV}, 10 \mathrm{eV}, 100 \mathrm{eV}, 1$ $\mathrm{keV}, 10 \mathrm{keV}, 100 \mathrm{keV}, 1 \mathrm{MeV}$, and $10 \mathrm{MeV}$.

What does neutron detection with gamma-detecting semiconductors gain us? First, neutron detection comes with little or no additional cost: neutrons are treated just like any other gamma ray. Second, the combined device is still a semiconductor, and as such is more compact than two individual detectors. As 
such, these kinds of devices are likely to find application in hand-held instrumentation and spaceflight, where power consumption, mass, and volume are at a premium.

Neutron-gamma discrimination in the $\mathrm{Cd}$ - and $\mathrm{Hg}$ - devices described above is accomplished by pulseheight analysis and its effectiveness depends on the resolution of the detector, and the incident gamma spectrum. The use of multiple independent gamma rays (such as by the addition of boron or other external absorbers) decreases the probability of false positive identification of neutrons because the multiple gamma rays must appear in specific relation to each other.

5 Conclusions The future of semiconductor neutron detectors is reasonably bright, although in the near term it will probably be limited to thermal neutron detection. There is active research in boronbased materials whose theoretical thermal neutron efficiencies can approach $100 \%$. However, the small volumes of these devices will make it necessary to construct large-area detectors by tiling. Using present-day technologies, such detectors will be financially impractical because of the cost of over US $\$ 1000 / \mathrm{cm}^{3}$ for finished material. Similar considerations apply to organic semiconductors.

Spectroscopy with semiconductor devices will probably not be economically feasible except with low efficiency in polyethylene-clad silicon (or other mass-produced semiconductor), boron-based materials, or with thermal detectors in Bonner sphere configurations. In the last case, however, the semiconductor is replacing a much less expensive scintillator or ${ }^{3} \mathrm{He}$ tube and is not a particularly economical substitution. However, boron-based semiconductors offer the possibility of resolution similar to that obtained with gamma rays (few per cent in room-temperature devices), but further research is needed to discover new materials amenable to mass production.

Cryogenic detectors can be expected to find applications in laboratory or other controlled settings for the detection of fast neutrons. The resolution offered by these devices is expected to enable the identification of neutron sources via analysis of the observed spectrum and perhaps the source's chemical form and identity of shielding material by observation of absorption and transmission resonances. Although not likely to become field instruments, the devices can be made transportable, and improvements in refrigeration techniques, such as the use of mechanical coolers in place of liquid nitrogen, pulse tubes in place of liquid helium, and a 2-stage adiabatic demagnitization refrigerator to bring the absorber to 0.1 $\mathrm{K}$, may well further that end.

From a materials point of view, although the ${ }^{6} \operatorname{Li}(\mathrm{n}, \alpha)$ reaction has a higher $Q$-value, the chemical instability (with respect to oxygen and water) of many lithium-containing compounds, the hazards associated with their handling, and their relatively low lithium content make boron-rich superconductors, insulators and semiconductors attractive. However, since many boron-rich materials (such as $\mathrm{MgB}_{2}$ ) are often not isotropic, not easily worked because they do not melt congruently, or, if they do, they do so only at high temperatures (making their single crystals or polycrystals difficult to grow, and leaving sintering the only alternative), it is not obvious that a satisfactorily large boron absorber is realizable. Nevertheless, since sintering is often possible, and the temperature sensor can be glued to the absorber with seemingly good results, the immense variety of boron-rich solids makes the search for suitable materials among them an exciting prospect.

Acknowledgments This work was supported by the U. S. Department of Energy, Office of Nonproliferation Research and Engineering (NA-22). The Oak Ridge National Laboratory is managed and operated for the U. S. Department of Energy by UT-Battelle, LLC, under contract DE-AC05-00OR22725. The Y-12 National Security Complex is managed and operated for the U. S. Department of Energy by BWXT Y-12, LLC, under contract DEAC05-00OR22800. Lawrence Livermore National Laboratory is managed and operated for the U. S. Department of Energy by the University of California under contract W-7405-Eng-48.

\section{References}

[1] M. F. Cunningham et al., Appl. Phys. Lett. 81, 159 (2002).

[2] J. E. Baciak and Z. He, Nucl. Instrum. Methods Phys. Res. A 505, 191 (2003). 
[3] A. Shor, Y. Eisen, and I. Mardor, IEEE Trans. Nucl. Sci. 51, 1204 (2004).

[4] P. G. Litovchenko et. al., Nucl. Instrum. Methods Phys. Res. A 518, 423 (2004).

[5] F. William Walker, Josef R. Parrington, and Frank Feiner, Nuclides and Isotopes, Fourteenth Edition (General Electric Company, San Jose, 1989), p. 92.

[6] T. M. Filho et al., Nucl. Instrum. Methods Phys. Res. A 458, 441 (2001).

[7] D. S. McGregor et al., Nucl. Instrum. Methods Phys. Res. A 380, 271 (1996).

[8] D. S. McGregor et al., IEEE Trans. Nucl. Sci. 49, 1999 (2002).

[9] T. Aoyama et al., Nucl. Instrum. Methods Phys. Res. A 314, 590 (1992).

[10] M. J. Knitel et al., Nucl. Instrum. Methods Phys. Res. A 443, 364 (2000).

[11] Z. W. Bell, G. M. Brown, C. H. Ho, and F. V. Sloop, Jr., Proc. SPIE, 4784, 150 (2002).

[12] M. Bowen et al., IEEE Trans. Nucl. Sci. 36, No. 1, 562 (1989).

[13] J. B. Birks, The Theory and Practice of Scintillation Counting (Pergamon, Oxford, 1964), chapters 3 and 6.

[14] Th. Förster, Ann. Phys. 2, 55 (1948).

[15] G. D. Scholes, Annu. Rev. Phys. Chem. 54, 57 (2003).

[16] B. W. Robertson et al., Appl. Phys. Lett. 80, 3644 (2002).

[17] A. N. Caruso et al., J. Phys.: Condens. Matter 16, L139 (2004).

[18] Z. W. Bell, U.S. Patent 6,011,266, 4 January 2000.

[19] R. T. Johnson, Jr., Nucl. Instrum. Meth. 77, 189 (1970).

[20] Z. W. Bell et al., U. S. Patent 6,544,442, 8 April 2003.

[21] G. F. Knoll, Radiation Detection and Measurement, Third Edition (Wiley, New York, 2000), pp. 748-751.

[22] S. H. Moseley, J. C. Mather, and D. McCammon, J. Appl. Phys. 56, 1257 (1984).

[23] P. de Marcillac et al., Nucl. Instrum. Methods Phys. Res. A 337, 95 (1993).

[24] C. S. Silver et al., Nucl. Instrum. Methods Phys. Res. A 485, 615 (2002).

[25] J. M. Richardson et al., Nucl. Instrum. Methods Phys. Res. A 306, 291 (1991).

[26] J. M. Richardson et al., IEEE Trans. Nucl. Sci. 45, 550 (1998).

[27] Z. Chowduri et al., Rev. Sci. Instrum. 74, 4280 (2003).

[28] K. Takahashi et al., Physica C 392-396, 1501 (2003).

[29] T. Nakamura et al., Nucl. Instrum. Methods Phys. Res. A 520, 67 (2004).

[30] T. Nakamura et al., Nucl. Instrum. Methods Phys. Res. A 520, 76 (2004).

[31] T. Niedermayr et al., Nucl. Instrum. Methods Phys. Res. A 520, 70 (2004).

[32] S. Friedrich et al., Nucl. Instrum. Methods Phys. Res. A 467-468, 1117 (2001).

[33] J. Castaing, R. Caudron, G. Toupance, and P. Costa, Solid State Commun. 7, 1453 (1969).

[34] E. F. Westrum and G. A. Clay, J. Chem. Thermo. 10, 629 (1978).

[35] C. E. Holcombe et al., High Temp. Sci. 5, 349 (1973).

[36] M. B. Chadwick and P. G. Young, ENDF/B-VI Mod 2 Evaluation, July 1997, available on-line from the National Nuclear Data Center, Brookhaven National Laboratory, www.nndc.bnl.gov.

[37] R. G. Miller and R. W. Kavanagh, Nucl. Instrum. Meth. 48, 13 (1967).

[38] S. Dési, Nucl. Instrum. Meth. 140, 467 (1977).

[39] C. Chung and Y. R. Chen, Nucl. Instrum. Methods Phys. Res. A 301, 328 (1991).

[40] S. F. Kozlov, E. A. Konorova, and I. A. Kuznetsov, IEEE Trans. Nucl. Sci. NS-24, 235 (1977).

[41] V. D. Kovalchuk, V. I. Trotsik, and V. D. Kovalchuk, Nucl. Instrum. Methods Phys. Res. A 351, 590 (1994).

[42] P. Bergonzo et al., Nucl. Instrum. Methods Phys. Res. A 476, 694 (2002).

[43] G. J. Schmid, J. A. Koch, R. A. Lerche, and M. J. Moran, Nucl. Instrum. Methods Phys. Res. A 527, 554 (2004).

[44] F. P. Doty, I. Zwieback, and W. Ruderman, U. S. Patent 6,388,260, 14 May 2002.

[45] F. P. Doty, U. S. Patent 6,727,504, 27 April 2004.

[46] F. P. Doty, C. M. Munoz, D. H. Morse, D. A. Chinn, and A. J. Anatolak, paper 5541-10, presented at SPIE Conference 5541, Penetrating Radiation Systems and Applications VI, Denver, Colorado, 4-5 August 2004.

[47] P. M. Siffert et al., U. S. Patent 3,999,071, 21 December 1976.

[48] A. G. Vradii, Atomnaya Energiya 42, 64 (1977).

[49] M. Fasasi, M. Jung, P. Siffert, and C. Teissier, Radiat. Prot. Dosim. 23, 429 (1988)

[50] D. S. McGregor, J. T. Lindsay, and R. W. Olsen, Nucl. Instrum. Methods Phys. Res. A 381, 498 (1996).

[51] A. G. Beyerle and K. L. Hull, Nucl. Instrum. Methods Phys. Res. A 256, 377 (1980).

[52] Z. W. Bell, K. R. Pohl, and L. van den Berg, IEEE Trans. Nucl. Sci. 51, 1163 (2004).

[53] F. William Walker, Josef R. Parrington, and Frank Feiner, op. cit., p. 42. 
[54] J. K. Tuli, Thermal Neutron Capture Gamma Rays, available on-line from the National Nuclear Data Center, Brookhaven National Laboratory, www.nndc.bnl.gov.

[55] G. F. Knoll, op. cit., pp. 539 - 545.

[56] MCNP - A General Monte Carlo N-Particle Transport Code, Version 5, available from the Radiation Safety Information Computational Center, Oak Ridge National Laboratory, Oak Ridge, TN, USA. 